\title{
Association Between Sleep Disorder and Increased Body Mass Index in Adult Patients
}

\author{
Bocicor Andreea Elena1, Buicu Gabriela1,2, Sabau Daniela1,2, Varga Andreea1,3, Tilea I1,3*, Gabos-Grecu I1,2 \\ 1 University of Medicine and Pharmacy Tirgu Mures, Romania \\ 2 Psychiatry I Clinic, County Hospital, Tirgu Mures, Romania \\ ${ }^{3}$ Cardiovascular Rehabilitation Clinic, Emergency County Hospital, Tirgu Mures, Romania
}

\begin{abstract}
Introduction and objectives. Obesity is a public health issue, with increasing prevalence and incidence all over the world. Diet and exercise applied in obesity treatment are not always as effective as expected, as there are many other determining factors which can lead to obesity. One of these modifiable factors seem to be sleep disorder. The objective of our study was to test the positive association between the presence of sleep disorder and increased body mass index (BMI). Material and method. 84 patients were screened in a descriptive cross-sectional study. Each patient completed the adjusted 7 items University of Toronto Sleep Assessment Questionnaire (SAQ@). Each affirmative answer was accounted 1 point. The total score was calculated. Mild sleep disorder was considered at 4-5 points, severe sleep disorder at 6 - 7 points. Body mass index (BMI) was calculated for each patient by the formula weight (Kg)/squared height $\left(\mathrm{m}^{2}\right)$. We considered increased BMl values greater than $25 \mathrm{~kg} / \mathrm{m} 2$. The association between the sleep disorder and increased BMI was statistically tested. Results. We interviewed 84 patients, 32 (38\%) men (average age 54 +/- 6.63) and 52 (62\%) women (average age 50 +/- 5.26). Mild sleep disorder was present in 36 patients, and severe sleep disorder in 25 patients. We noticed association between sleep disorder and increased BMI $(p=0.0064, R R=2.925,95 \% \mathrm{Cl} 1.16-7.36)$. We observed the risk for increased BMl dependent on the sleep disorder severity. Conclusions. Sleep disorder is a potentially modifiable risk factor which can be included in obesity therapeutic approach and management. Early diagnosis and treatment of sleep disorder is important in obesity prevention.
\end{abstract}

Keywords: Sleep disorder, increased body mass index, obesity, risk, prevention.

Received: 22 December 2015 / Accepted: 12 March 2016

\section{Introduction and objectives}

Sleep disorder, especially short duration of sleep and modification in sleep quality, can result in neurocognitive and hormonal changes that influence health status of the affected individuals. A large number of comparative studies demonstrated that in concert with modifications in sleep duration and quality there has been an increase in the prevalence of obesity [1]. Obesity and overweight, expressed by increased body mass index (BMI) are public health issues, with increasing prevalence and incidence all over the world. Obesity also represents an important risk for other diseases [1,2]. Although obesity seem to be the result of excessive consumption of calories combined with insufficient or lack of physical activity, there are several other determining factors contributing to excessive adiposity [3]. One of these factors seem to be sleep disorder, the modifications in sleep duration and quality being associated with an increase in adiposity and body weight [4]. Diet and exercise applied in obesity treatment are not always as effective as expected, as there are many other determining factors which can lead to obesity. Several studies have tested the association between sleep disorder and obesity, and most of these studies demonstrated the association between short sleep duration and increased body mass index, although some of them claimed that this association is not

* Correspondence to: Ioan Tilea

E-mail: ioan.tilea@umftgm.ro very significant in adults and older adults [5]. According to other studies, modification in sleep duration is associated not only to increased body mass index but to increased waist circumference. These findings have proven that short sleepers are more likely to be obese and have abdominal adiposity, and this morbid association does not depend on gender, age or ethnicity [6].

Research prove that an adequate sleep duration and quality plays an important role in weight management and decreasing the risk of metabolic disorders. Both sleep quality and normal weight are important for short and longterm health. Therefore, lifestyle assessment in any overweight or obese patient must include sleep evaluation as restoring normal sleep can improve diet and exercise results in obese patients $[4,6]$.

The objective of our study was to test the positive association between the presence of sleep disorder and increased body mass index (BMI).

\section{Material and method}

84 patients, aged 18 to 65 years old, were screened in a descriptive cross-sectional study. The main objective of the study was to test the positive association between the presence of sleep disorder and increased body mass index. The presence of sleep apnea, decompensated chronic diseases and working night shifts were sample exclusion criteria. For this study we considered the most prevalent sleep disorder characterized by criteria that could be assessed with 
a standardized sleep questionnaire. These criteria were: difficulties to fall asleep, shortness in sleep duration, the quality of sleep (repeated awakenings, restless sleep and/or presence of nightmares, earlier awakening, tiredness during the day) and sleep inductors drugs addiction. To assess these criteria each patient completed the adjusted 7 items University of Toronto Sleep Assessment Questionnaire (SAQ) (Appendix). Each affirmative answer was accounted 1 point. The total score of each completed questionnaire was calculated. We considered mild sleep disorder present at a score of 4-5 points, respectively severe sleep disorder was considered at 6-7 points. Body mass index (BMI) was calculated for each patient by the formula weight $(\mathrm{Kg}) /$ squared height $\left(\mathrm{m}^{2}\right)$. We considered increased BMI values greater than $25 \mathrm{~kg} / \mathrm{m}^{2}$. The association between the sleep disorder and increased BMI was statistically tested using Chi square Fischer exact test.

\section{Results}

We interviewed 84 adult patients admitted in a cardiovascular rehabilitation clinic, $32(38 \%)$ men (average age 54 $+/-6.63$ ) and 52 (62\%) women (average age 50+/-5.26). Sleep disorder was identified in 61 patients at a score greater than or equal to 4 points in SAQ. 36 patients presented mild sleep disorder (SAQ score 4 or 5) and severe sleep disorder was present in 25 patients (SAQ score 6 or 7).

According to SAQ answers given by the 61 patients with sleep disorder we noticed 54 patients with difficulties in falling asleep, 42 patients with sleep duration less than 5 hours, 37 patients presenting both mentioned sleep disorder characteristics. There were also 23 patients with affirmative answers to all 3 questions assessing sleep quality (frequent awakenings during the night, frequent nightmares and early awakenings followed by daytime sleepiness). We also mention that in 61 patients identified with mild or severe sleep disorder 48 found impossible to fall asleep without medication.

We noticed statistically significant association between the presence of sleep disorder (SAQ score greater or equal to 4 ) and increased BMI ( $\mathrm{p}=0.0064, \mathrm{RR}=2.925,95 \% \mathrm{CI}$ 1.16-7.36) as shown in table I.

The association between sleep disorder and increased BMI was also tested for sleep disorder degrees of severity. The association between mild sleep disorder (SAQ score 4-5) and increased BMI was present, but not quite statistically significant $(\mathrm{p}=0.0482, \mathrm{RR}=2.56,95 \%$ CI $0.98-6.69)$ as presented in table II.

We found the association between severe form of sleep disorder (SAQ score 6-7) and increased BMI strongly statistically significant $(\mathrm{p}=0.0034, \mathrm{RR}=3.45,95 \%$ CI 1.34 8.89), as presented in Table III.

\section{Discussion}

University of Toronto SAQ, initially designed to identify sleep disorder alone or in relationship with sleep apnea, has been modified or simplified according to the purpose
Table I. Overall association between sleep disorder (SAQ score $\geq$ 4) and increased BMI

\begin{tabular}{lccc}
\hline SAQ score & $\mathrm{BMI} \geq 25 \mathrm{Kg} / \mathrm{m} 2$ & $\mathrm{BMI}<25 \mathrm{Kg} / \mathrm{m} 2$ & Total \\
\hline Increased $(\geq 4)$ & 31 & 30 & 52 \\
Normal $(0-3)$ & 4 & 19 & 23 \\
Total & 35 & 49 & 84 \\
\hline
\end{tabular}

$\mathrm{p}=0.0064, \mathrm{RR} 2.92,95 \% \mathrm{Cl} 1.15-7.36$

Table II. Association between mild sleep disorder (SAQ score 4-5) and increased BMI

\begin{tabular}{lccc}
\hline SAQ score & $\mathrm{BMI} \geq 25 \mathrm{Kg} / \mathrm{m}^{2}$ & $\mathrm{BMI}<25 \mathrm{Kg} / \mathrm{m}^{2}$ & Total \\
\hline Mild increased (4-5) & 16 & 20 & 36 \\
Normal $(0-3)$ & 4 & 19 & 23 \\
Total & 20 & 39 & 59 \\
\hline
\end{tabular}

$\mathrm{p}=0.0482, \mathrm{RR} 2.56,95 \% \mathrm{Cl} 0.98-6.69$

Table III. Association between severe sleep disorder (SAQ score 6-7) and increased BMI

\begin{tabular}{lccc}
\hline SAQ score & $\mathrm{BMI} \geq 25 \mathrm{Kg} / \mathrm{m} 2$ & $\mathrm{BMI}<25 \mathrm{Kg} / \mathrm{m} 2$ & Total \\
\hline Severe increased $(6-7)$ & 15 & 10 & 25 \\
Normal $(0-3)$ & 4 & 19 & 23 \\
Total & 19 & 29 & 48 \\
\hline $\mathrm{p}=0.034$, RR 3.45, 95\% Cl $1.34-8.89$ & &
\end{tabular}

of several studies proving the association between sleep disorders and other co-morbidities [5-7]. Our study did not take into account the presence of sleep apnea and restless legs syndrome, therefore we used the adjusted 7 items SAQ in order to estimate sleep duration, quality and influence of sleep medication as main parameters to detect chronic uncomplicated primary sleep disorder and its degrees of severity.

In our interviewed 84 adult patients, $61(72.6 \%)$ were found with sleep disorder, 25 patients (29.7\%) presenting severe, persistent forms of insomnia. Our results correspond to general European and Northern American adult population trends [8] with a sleep disorder prevalence situated between 28 and $31 \%$ for the age group of 50 years old and older $[8,9]$. Sleep disorder affects approximately $1 / 3$ of adult population annually and seem to be a significant cause of morbidity and mortality [9]. The prevalence, the health impact, the morbid associations and management of sleep disorder tend to be overlooked. This underappreciation and undertreatment of sleep disorder, make this group of illnesses an increasing health concern [8-11].

According to data presented in table I we found that the overall association between sleep disorder and increased $\mathrm{BMI}$ is statistically significant $(\mathrm{p}=0.0064, \mathrm{RR}=2.925,95 \%$ CI 1.16-7.36). Our findings confirm the results of several other studies that the presence of chronic sleep disturbances seem to be an important contributing risk factor to overweight and obesity onset [4-6,12]. Most of those studies established the association between sleep disorder and obesity by taking into account certain parameters like sleep duration or quality of sleep, only a few of them using combined data characteristic to sleep disorder [4-6]. Divided by degrees of sleep disorder severity assessed by SAQ accumulated number of total points, our data showed there is an association between mild form of sleep disorder 
and increased BMI, but the association is not quite statistically significant $(\mathrm{p}=0.0482, \mathrm{RR}=2.56,95 \%$ CI 0.98 6.69). Instead we found strongly significant association $(\mathrm{p}=0.0034, \mathrm{RR}=3.45,95 \%$ CI 1.34-8.89) between severe sleep disorder and increased BMI. The association between the sleep disorder and increased BMI tends to be stronger for severe forms than in mild forms of disease, suggesting it may depend on sleep disorder severity (number of characteristic symptoms experienced by individuals during the same time unit). Considering this, our findings match other studies testing the association between severe chronic sleep disorder and obesity, pointing the fact that the more severe sleep disorder, most likely the patients associate increased BMI [4,5,12-14]. Moreover, some of these studies were long time surveys which suggest a curvilinear relationship between sleep disorder and obesity [12-14]. Many researches confirm that the most frequent characteristic of sleep disorder leading to obesity is short sleep duration. These data also match our findings: 42 of 61 patients with sleep disorder were short sleepers, 54 of 61 experienced difficulties in falling asleep, and only 23 of 61 patients experienced symptoms characteristic to sleep quality alteration [5,15-17]. Several studies emphasize the fact that the earlier onset of sleep disturbances, like in childhood or infancy, the more likely the adolescents or young adults are exposed to obesity [17-20]. The idea generated by these studies suggest more prospective surveys are required on larger number of younger patients and on children in order to early identify and correct sleep disorder as part of obesity primary prevention program.
Originality of our research is that we used combined data characteristic to sleep disorder, as SAQ answers concerning both sleep duration and quality were analyzed together.

Most likely, sleep disorder and increased BMI are associated due to neuro-hormonal mechanisms, initially triggered by sleep deprivation $[5,9,18,19]$. The connections between the two phenomena, sleep disorder and obesity expressed by increased BMI, are multiple and yet to be investigated in furthermore studies.

\section{Conclusions}

Sleep disorder is an important and potentially modifiable risk factor contributing to increased body mass index. Sleep assessment questionnaires easily adjustable according to patients and purposes of studies can be successfully used to identify uncomplicated primary sleep disorders. Assessment of sleep disorder presence must be included in obesity therapeutic approach and management. Early diagnosis and treatment of sleep disorder is important in obesity prevention, as an affordable and easy accessible part of lifestyle changes in overweight and obesity prevention and treatment management strategy.

\section{Conflict of interest}

None to declare

\section{References}

1. Vorona RD, Winn Maria, Babineau Teresa, et al. Overweight and Obese Patients in a Primary Care Population Report Less Sleep Than Patients with a Normal Body Mass Index. Arch Intern Med. 2005;165(1):25-30.

Appendix: Sleep Assessment Questionnaire $\odot$ (SAQ)

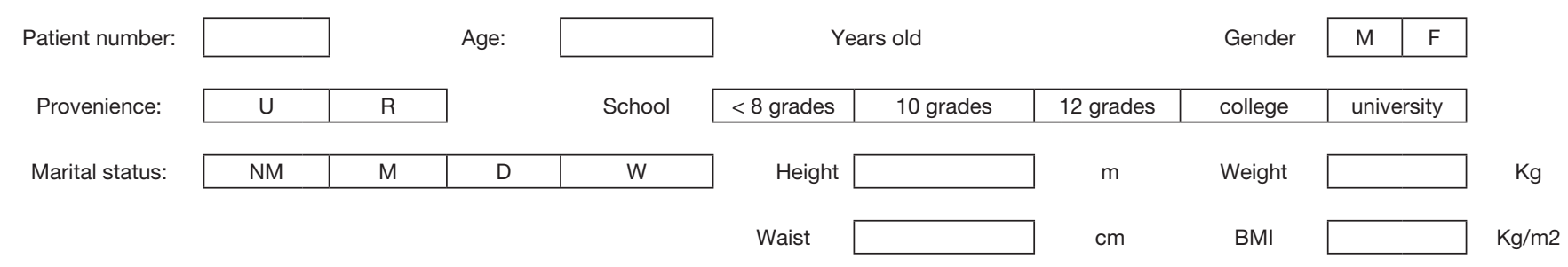

PLEASE ANSWER EACH QUESTION BY CHECKING THE ONE ANSWER THAT FITS BEST At least over the past month, how often have you experienced the following:

\begin{tabular}{|l|l|}
\multicolumn{1}{c}{ At least over the past month, how often have you experienced the following: } & \multicolumn{1}{c}{ NOS } \\
\hline 1. Difficulty falling asleep (does it take longer than 30 minutes)? & 1 point \\
\hline 2. Sleeping for less than 5 hours? & \\
\hline 3. Repeated awakenings (more than 3) during your sleep? & \\
\hline 4. Nightmares or agitated sleep? & \\
\hline 5. Waking up before you want to (i.e., getting less sleep than you need)? & \\
\hline 6. Waking up NOT feeling refreshed or thoroughly rested? & \\
\hline 7. Frequently using medication for sleep or nervousness? & \\
\hline Total points & \\
\hline
\end{tabular}


2. Caballero B. The global epidemic of obesity: An overview. Epidemiol Rev. 2007;29:1-5.

3. James WP. The fundamental drivers of the obesity epidemic. Obes Rev. 2007;9(Suppl1):6-13.

4. Hargens TA, Kaleth AS, Edwards Elizabeth, Butner Katrina. Association between sleep disorders, obesity, and exercise: a review. Nat Sci Sleep. 2013;5:27-35

5. Ford ES, Li Chaoyang, Wheaton Anne, et al. Sleep Duration and Body Mass Index and Waist Circumference among US Adult. Obesity. 2014;22(2):598-606

6. Kondracki Nancy L. The Link Between Sleep and Weight Gain, Today's Dietitian, 2012;14(6):48

7. Cesta A, Moldofsky H, Sammut C. The University of Toronto Sleep Assessment Questionnaire@ (SAQC). Sleep Res. 1996;25:486.

8. Ferrie Jane E, Kumari Meena, Salo Paula,et al. Sleep epidemiology-a rapidly growing field. Int J Epidemiol. 2011;40(6):1431-1437.

9. American Psychiatric Association. Diagnostic and Statistical Manual of Mental Disorders, 4th Ed, Text Revision (DSM-IV-TR). Washington, DC: American Psychiatric Association 2000.

10. Hossain JL, Shapiro CM. The prevalence, cost implications, and management of sleep disorders: an overview, Sleep Breath. 2002;6(2):85102.

11. Ram S, Seirawan H, Kumar SKS, Clark GT. Prevalence and impact of sleep disorders and sleep habits in the United States. Sleep and
Breathing. 2010;14(1):63-70.

12. Taheri S, Ling Lin, Diane Austin, Young T, Mignot E. Short Sleep Duration Is Associated with Reduced Leptin, Elevated Ghrelin, and Increased Body Mass Index. PLoS Medicine. 2004;1(3):210-216.

13. Gangwisch EJ, Dolores Malaspina, Bernadette Boden-Albala, Heymsfield SB. Inadequate Sleep as a Risk Factor for Obesity: Analyses of the NHANES I. Sleep, 2004;28(10):1289-1295.

14. Girardin JL, Natasha J Williams, Sarpong D, et al. Associations Between Inadequate Sleep and Obesity in the US Adult Population. BMC Public Health. 2014;14(290).

15. Wu Y, Zhai L, Zhang D. Sleep duration and obesity among adults: a metaanalysis of prospective studies, Sleep Med. 2014;15(12):1456-62.

16. Patel SR, Hu FB. Short sleep duration and weight gain: a systematic review. Obesity (Silver Spring), 2008;16:643-53.

17. Nielsen LS, Danielsen KV, Sorensen TI. Short sleep duration as a possible cause of obesity: critical analysis of the epidemiological evidence. Obes Rev. 2011;12:78-92.

18. Reilly JJ, Armstrong J, Dorosty AR, et al. Early life risk factors for obesity in childhood: cohort study. BMJ 2005;330:1357.

19. Taveras EM, Rifas-Shiman SL, Oken E, et al.Short sleep duration in infancy and risk of childhood overweight. Arch Pediatr Adolesc Med. 2008;162:305-11.

20. Paul IM, Savage JS, Anzman SL, et al., Preventing obesity during infancy: a pilot study. Obesity (Silver Spring), 2011;19:353-61. 